\title{
Acute syphilitic transverse myelitis: unusual presentation of meningovascular syphilis
}

\author{
DANIEL H LOWENSTEIN,* CATHERINE MILLS, $\dagger$ ROGER P SIMON* \\ From the Departments of *Neurology and $\dagger$ Neuroradiology, University of California, San Francisco, California, \\ United States of America
}

SUMMARY A case of meningovascular syphilis presenting with the rare spinal cord manifestations is reported. The angiographic, computed tomographic, and magnetic resonance images of the subsequent cerebrovascular lesions are shown. The presentation, evolution, and treatment of this unusual form of vascular syphilis are discussed.

Before antibiotics were discovered acute syphilitic transverse myelitis was a well recognised but unusual manifestation of infection with Treponema pallidum. ${ }^{1}$ Since that time, with the advent of effective diagnosis and treatment, the overall incidence of neurosyphilis in any form has fallen dramatically. In recent years, however, the number of cases of primary syphilis has again increased. ${ }^{2}$ With this has come the call for renewed vigilance over the neurological expressions of this disease. ${ }^{3}$

To emphasise its clinical variety, we report an unusual presentation of meningovascular syphilis in a young homosexual man.

\section{Case report}

A homosexual man aged 26 presented to San Francisco General Hospital reporting several hours of leg weakness and inability to walk. He had had no prodromal symptoms except for an episode of gastroenteritis with nausea and vomiting two to three weeks earlier and a persistent mild upper respiratory infection. On the night before admission he had drunk a moderate amount of alcohol at a party. When he awoke the next morning he noticed that his right leg was weak, and he stumbled when attempting to walk. He had no other weakness, sensory abnormalities, back pain or tenderness, or changes in sphincter function.

Address for reprints: Dr Roger P Simon, Department of Neurology, 4M62, San Francisco General Hospital, 1001 Potrero Avenue, San Francisco, California 94110 , USA

Accepted for publication 13 January 1987
He reported having had multiple homosexual relationships, though not with men known to have the acquired immune deficiency syndrome (AIDS). He recalled being treated for syphilis with an intramuscular injection of penicillin at least once in the past, possibly as recently as six months before admission to hospital. His medical records were not available.

On examination, his temperature was $36.7^{\circ} \mathrm{C}$, pulse 88 a minute and regular, blood pressure 124/70 $\mathrm{mm} \mathrm{Hg}$, and respirations 16 a minute. General examination showed no adenopathy, meningismus, spinal tenderness, or other abnormalities. His mental status appeared to be normal, although he was somewhat indifferent and unable or unwilling to provide further details about his medical past. Cranial nerve examination showed no abnormality. Motor examination showed increased tone and moderate pyramidal weakness in both legs, worse on the right and more severe distally. The leg reflexes were hyperactive, with sustained clonus at the ankles, and the plantar responses were extensor bilaterally. There was pronounced decrease in rectal sphincter tone and no anal wink. The cremasteric and abdominal reflexes were absent on the right and present on the left. The patient was unable to walk because of weakness. The sensory examination was entirely normal.

Initial routine laboratory investigations showed normal complete blood count, serum electrolytes, renal and liver function tests, and urine analysis. Computed tomography of the head gave normal results. A thoracic myelogram on the night of admission was notable only for mild degenerative disc disease. Analysis of the cerebrospinal fluid (CSF) showed glucose concentration of $1.9 \mathrm{mmol} / 1$ (simulta- 


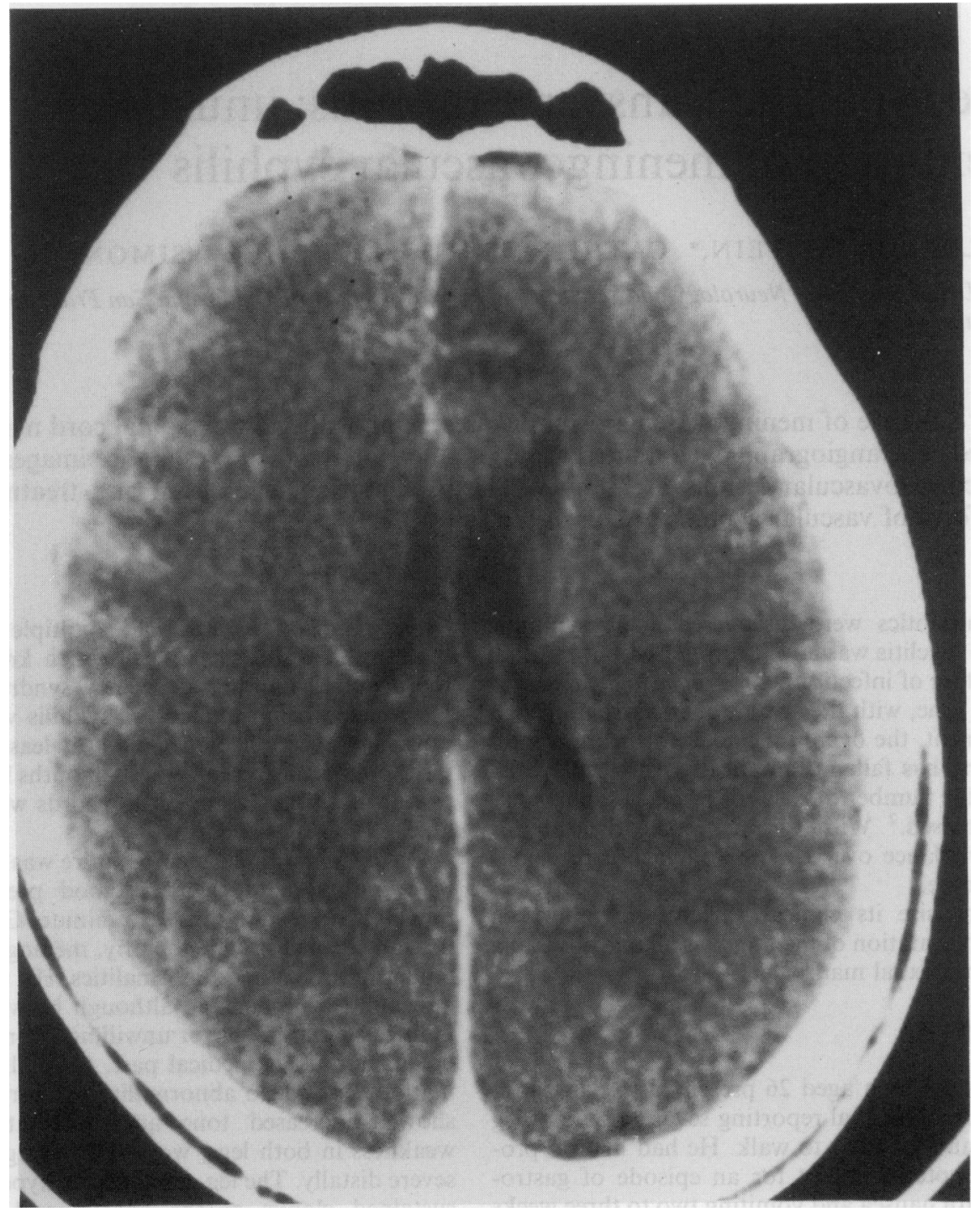

Fig 1 Computed tomograph showing low attenuation in periventricular white matter on right and in left medial frontal lobe.

neous blood glucose of $4.4 \mathrm{mmol} / \mathrm{l})$, protein concentration of $1.20 \mathrm{~g} / \mathrm{l}$, erythrocyte count $15 \times 10^{6} / 1$ and white cell count $12 \times 10^{6} / 1$, with a differential count of $83 \%$ lymphocytes and $17 \%$ monocytes. The CSF had an IgG index of 5.0 (normal <0.6) and was positive for oligoclonal bands. The CSF gave negative results for cryptococcal antigen, and no growth was observed later on cultures for bacteria or fungi.

By the following morning, the patient's weakness had improved noticeably without specific treatment, though he continued to have spasticity in the legs. On the third hospital day he appeared to be sleepy and had a mild cognitive deficit. A repeat lumbar puncture showed an opening pressure of $2.52 \mathrm{kPa}(210$ $\mathrm{mm} \mathrm{H}_{2} \mathrm{O}$ ) and yielded clear, colourless fluid with the following values: glucose concentration $2.0 \mathrm{mmol} / \mathrm{l}$, protein concentration $0.98 \mathrm{~g} / 1$, erythrocyte count $6 \times$ $10^{6} / 1$, white cell count $130 \times 10^{6} / 1$, with $92 \%$ lymphocytes and $8 \%$ monocytes.

On the fourth hospital day the patient developed 


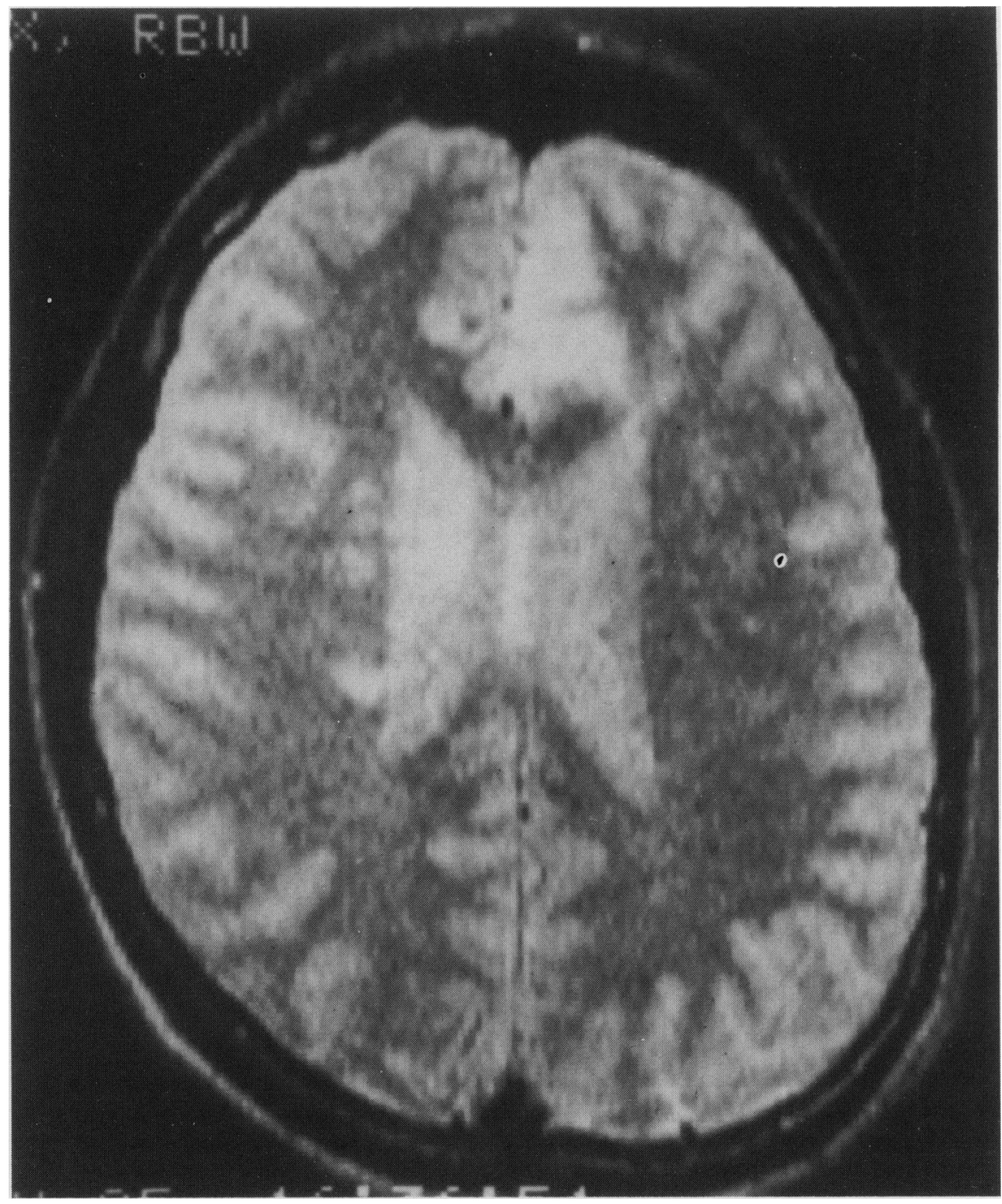

Fig 2 Magnetic resonance image obtained with TR of 3000 msec and TE of 64 msec showing multiple areas of abnormal high signal intensity in periventricular white matter, body of right caudate nucleus, and left medial frontal lobe.

mild face and arm weakness on the left side. The result of the Venereal Disease Research Laboratory (VDRL) test on serum taken at the time of admission was positive at a dilution of 1:32. A repeat head computed tomography scan showed interval development of right hemispheric white matter oedema with evidence of mass effect, as well as a focal low attenuation lesion in the left frontal lobe (figure 1). An electroencephalogram showed diffuse symmetrical slow activity. On the fifth hospital day, with the presumptive diagnosis of neurosyphilis, the patient was treated intravenously with $2.5 \mathrm{MIU}$ aqueous benzyl penicillin every four hours. On the following day the result of the VDRL test on CSF obtained on admission was reported as positive at a dilution of $1: 2$.

The patient continued to deteriorate and, because of his risk factors, concern was raised about other neurological manifestations of AIDS. He therefore underwent a magnetic resonance scan and a cerebral angiogram. The magnetic resonance image (figure 2) showed multiple high intensity lesions in the periventricular white matter on the left, the body of the 


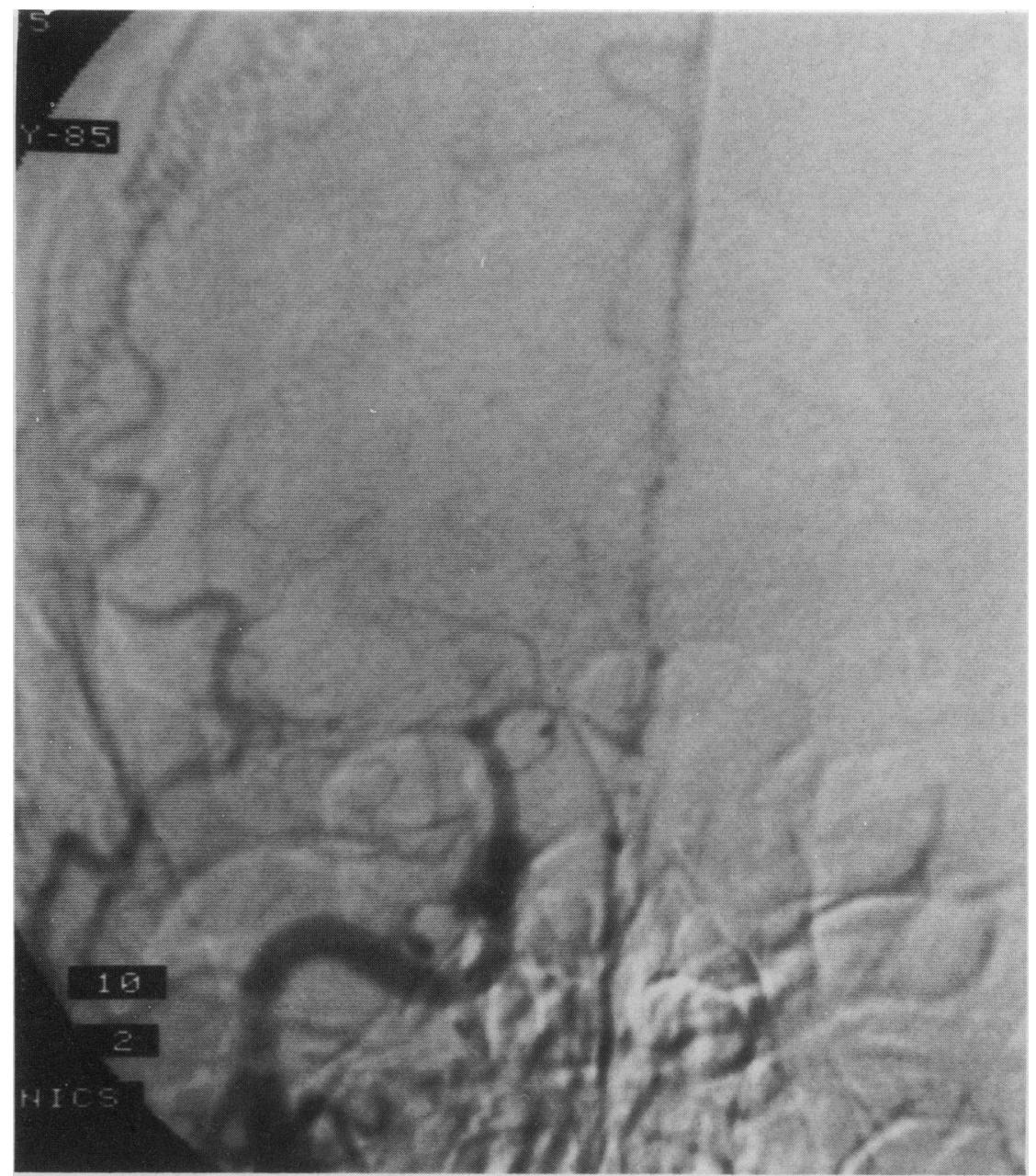

Fig 3 Right common carotid arteriogram showing pronounced narrowing of MI segment of middle cerebral artery and irregular narrowing of $A 1$ segment of anterior cerebral artery and pericallosal artery, characteristic of vasculitis.

right caudate nucleus, the right lentiform nucleus, and the left frontal and parietal lobes. The angiogram (figure 3) showed extreme narrowing with near occlusion of the right middle cerebral artery at the M1 segment. Luminal contour irregularities were also seen in the right and left $\mathrm{Al}$ segments of the anterior cerebral arteries, the left M1 segment of the middle cerebral artery, and several middle artery branches, as well as diffuse vasculitic changes of many medium calibre vessels (figure 4). These changes further supported the diagnosis of meningovascular syphilis.

The patient continued on a 14 day course of intravenous penicillin treatment. His mild encephalopathy, left hemiparesis, and spastic paraparesis slowly improved. By the time of his discharge, his mental state and strength were back to his normal baseline, he walked with a slightly broad base, and had persistent hyperreflexia in the legs. CSF analysis at the completion of treatment showed: glucose concentration $2.4 \mathrm{mmol} / 1$, protein concentration $0.72 \mathrm{~g} / \mathrm{l}$, erythrocyte count $26 \times 10^{6} / 1$, white cell count $67 \times$ $10^{6} / 1$, with $87 \%$ monocytes and $13 \%$ lymphocytes.

\section{Discussion}

Bastian, in the 1882 edition of Quain's dictionary of medicine, was apparently the first to suggest that the softening of the spinal cord observed in patients with syphilis was due to thrombosis of spinal vessels. ${ }^{4}$ The further elucidation of a spinal form of meningo- 


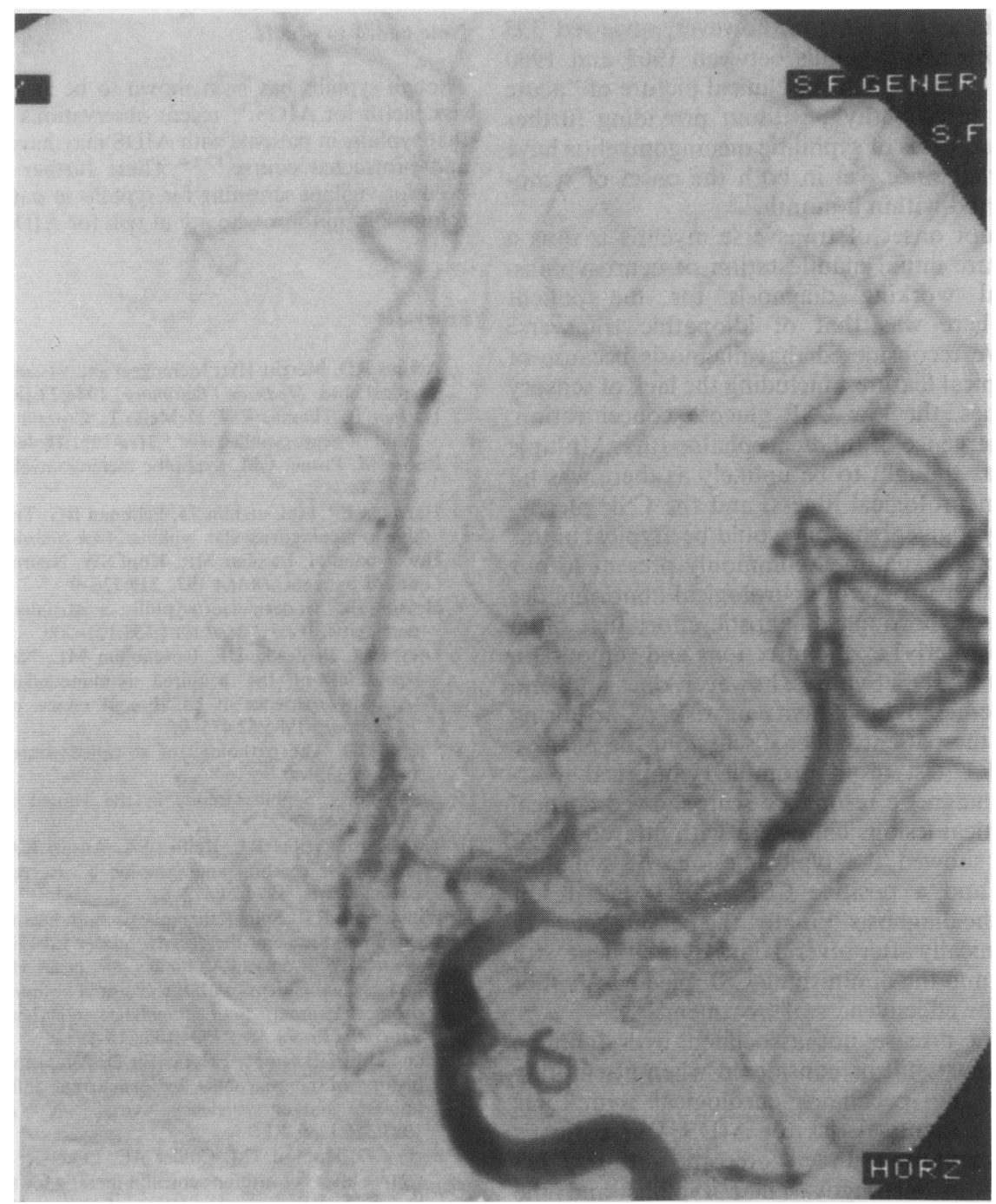

Fig 4 Left common carotid arteriogram showing luminal narrowing of Al segment, MI segment, and several middle cerebral artery branches, compatible with vasculitis.

vascular syphilis was made in 1894 by Williamson, who, in the introduction to a case report with postmortem study, remarked that "from the frequency and importance of cerebral thrombosis one would expect that thrombosis in the spinal vessels would also play an important part in the diseases of the spinal cord." 5 In 1902, Singer advanced this notion in a review of 15 patients with acute myelitis associated with syphilis. The two patients presented in some detail had an abrupt onset of symptoms (several hours and two to three days, respectively), and necropsy showed syphilitic arteritis with thrombosis of the spinal vessels. ${ }^{4}$
In their classic 1944 treatise, Adams and Merritt discussed extensively the various spinal syndromes associated with syphilis, apart from tabes dorsalis. ${ }^{1}$ Among their patients were 10 with "acute syphilitic transverse myelitis", characterised by the acute onset of paraplegia, loss of sphincter control, and sensory loss. As before, the presumed aetiology was abrupt thrombosis of a spinal artery due to syphilitic endarteritis. They noted that this entity was rare, accounting for only $1.5 \%$ of their series of 676 patients with neurosyphilis.

No mention of acute transverse myelitis was made in Hooshmand's review of 241 cases of neurosyphilis 
published in $1972 .{ }^{6}$ Hotson, however, reviewed 335 patients with neurosyphilis between 1965 and 1980 and noted 10 cases with the clinical picture of "acute or chronic myelopathy", without providing further details. ${ }^{3}$ Two cases of syphilitic meningomyelitis have been reported since, yet in both the onset of symptoms occurred within a month. ${ }^{78}$

The abrupt onset of transverse myelitis is thus a distinctly rare initial manifestation of neurosyphilis. Our initial working diagnosis for the patient presented here was that of idiopathic transverse myelitis. We reconsidered that diagnosis because of several atypical features, including the lack of sensory abnormalities, the low CSF glucose concentration, and the emergence of mild encephalopathy. Multiple sclerosis was thought to be unlikely as there was no history of neurological illness, and the CSF pleocytosis and hypoglycorrhachia would be atypical in this disease. Homosexual men commonly present to our hospital with a variety of neurological abnormalities and require a thorough diagnostic effort that often discloses (formerly) exotic infections and tumours in the setting of AIDS. Syphilis, however, does not seem to have increased in patients at risk of acquiring AIDS. ${ }^{9}$ In the patient under discussion, the positive serum VDRL test result prompted empirical treatment. The magnetic resonance scan showed cortical and subcortical lesions consistent with infarction, an angiogram showed the typical lesions of syphilitic arteritis, ${ }^{10}$ and a positive CSF VDRL test result confirmed the diagnosis. Finally, the patient began to improve clinically after several days of treatment with antibiotics, and the diminishing CSF pleocytosis substantiated the effectiveness of treatment. ${ }^{11}$

Syphilis, a disease not associated with immune deficiency, must still be considered when attempting to explain the peculiar neurological symptoms observed in patients at risk for AIDS. Our patient's unusual presentation underscores the diffuse CNS involvement in meningovascular syphilis and the reversibility of this protean disease.

\section{Note added to proofs}

Though syphilis has been shown to be an epidemiological risk factor for AIDS, ${ }^{12}$ recent observations have suggested that syphilis in patients with AIDS may have a more severe and protracted course. ${ }^{1314}$ These further emphasise the need for vigilant screening for syphilis in patients with neurological symptoms who are at risk for AIDS.

\section{References}

1 Adams RD, Merritt HH. Meningeal and vascular syphilis of the spinal cord. Medicine (Baltimore) 1944;23:181-214.

2 Dattner B, Thomas EW, DeMello L. Criteria for the management of neurosyphilis. Am J Med 1951;10:464-7.

3 Fisher M, Posner CM. Syphilitic meningomyelitis. Arch Neurol 1977;34:785.

4 Harrigan EP, McLaughlin TJ, Feldman RG. Transverse myelitis due to meningovascular syphilis. Arch Neurol 1984;41:337-8.

5 Hooshmand H, Escobar MR, Kopf SW. Neurosyphilis: a study of 241 patients. JAMA 1972;219:726-9.

6 Hotson JR. Modern neurosyphilis: a partially treated chronic meningitis. West J Med 1981;135:191-200.

7 Levy RM, Bredeson DE, Rosenblum ML. Neurological manifestations of the acquired immunodeficiency syndrome (AIDS): experience at UCSF and review of the literature. J Neurosurg 1985;62:475-95.

8 Singer HD. The pathology of so-called acute myelitis. Brain 1902;25:332-40.

9 Anonymous. Syphilis trends in the United States. MMWR 1981;30:441-9.

10 Vatz KA, Scheibel RL, Keiffer SA, Ansari KA. Neurosyphilis and diffuse cerebral angiography: a case report. Neurology (Minneapolis) 1974;24:472-6.

11 Williamson RT. Spinal thrombosis and haemorrhage due to syphilitic disease of the vessels. Lancet 1894;ii:14-6.

12 Guinan ME, Thomas PA, Pinsky PF, et al. Heterosexual and homosexual patients with the acquired immunodeficiency syndrome: a comparison of surveillance, interview and laboratory data. Ann Intern Med 1984;100:213-8.

13 Johns DR, Tierney M, Felsenstein D. Alteration in the natural history of neurosyphilis by concurrent infection with the human immunodeficiency virus. $N$ Engl $J$ Med 1987;316:1569-72.

14 Berry CD, Hooton TM, Collier AC, Lukehart SA. Neurologic relapse after benzathine penicillin therapy for secondary syphilis in a patient with HIV infection. $N$ Engl $J$ Med 1987;317:1587-9. 